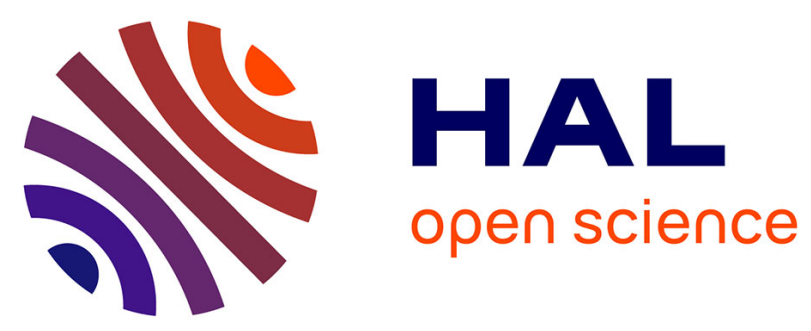

\title{
Étude expérimentale et théorique de la production de nuclides légers rapides dans les interactions proton-noyau à haute énergie
}

J-P. Alard, L. Avan, M. Avan, A. Baldit, J.P. Costilhes, A. Cordaillat, J. Dhermain, L. Fraysse, J. Fargeix, G. Roche, et al.

\section{To cite this version:}

J-P. Alard, L. Avan, M. Avan, A. Baldit, J.P. Costilhes, et al.. Étude expérimentale et théorique de la production de nuclides légers rapides dans les interactions proton-noyau à haute énergie. Journal de Physique, 1972, 33 (5-6), pp.477-483. 10.1051/jphys:01972003305-6047700 . jpa-00207274

\section{HAL Id: jpa-00207274 https://hal.science/jpa-00207274}

Submitted on 1 Jan 1972

HAL is a multi-disciplinary open access archive for the deposit and dissemination of scientific research documents, whether they are published or not. The documents may come from teaching and research institutions in France or abroad, or from public or private research centers.
L'archive ouverte pluridisciplinaire HAL, est destinée au dépôt et à la diffusion de documents scientifiques de niveau recherche, publiés ou non, émanant des établissements d'enseignement et de recherche français ou étrangers, des laboratoires publics ou privés. 
Classification

Physics Abstracts

13.42

\title{
ÉTUDE EXPÉRIMENTALE ET THÉORIQUE DE LA PRODUCTION DE NUCLIDES LÉGERS RAPIDES DANS LES INTERACTIONS PROTON-NOYAU A HAUTE ÉNERGIE
}

\author{
J. P. AlARD, L. AVAN, M. AVAN, A. BALDIT, J. P. COSTILHES, A. CORDAILLAT (*),
} J. DHERMAIN, L. FRAYSSE, J. FARGEIX, G. ROCHE, J.-C. TAMAIN

Laboratoire de Physique Nucléaire, UER Sciences Exactes et Naturelles Université de Clermont-Ferrand

(Reçu le 23 décembre 1971)

\begin{abstract}
Résumé. - Nous donnons la description d'une expérience réalisée au CERN et concernant la production de $1 \mathbf{H},{ }^{2} \mathrm{H},{ }^{3} \mathrm{H},{ }^{3} \mathrm{He},{ }^{4} \mathrm{He}$ lors des interactions proton-noyau $\left({ }^{12} \mathrm{C},{ }^{27} \mathrm{Al},{ }^{197} \mathrm{Au}\right)$ à des angles variés $\left(30^{\circ}, 45^{\circ}, 75^{\circ}, 105^{\circ}\right)$. Nous présentons également une théorie thermodynamique permettant d'interpréter les résultats expérimentaux obtenus.

Abstract. - We give the description of an experiment performed at CERN concerning the production of $1 \mathrm{H},{ }^{2} \mathrm{H},{ }^{3} \mathrm{H},{ }^{3} \mathrm{He},{ }^{4} \mathrm{He}$ in proton-nucleus interactions (targets $\left.:{ }^{12} \mathrm{C},{ }^{27} \mathrm{Al},{ }^{197} \mathrm{Au}\right)$ at various angles $\left(30^{\circ}, 45^{\circ}, 75^{\circ}, 105^{\circ}\right)$. We also present a thermodynamical theory which permits an interpretation of our experimental results.
\end{abstract}

I. Introduction. - Le programme expérimental «SC 33 » accepté par le comité de Physique III du CERN [1] nous permet d'effectuer une étude de la fragmentation des noyaux bombardés par des protons de $600 \mathrm{MeV}$.

Nous donnons dans le tableau I quelques exemples

TABLEAU I

\begin{abstract}
Auteurs
P. A. Piroue, A. J. S.

Smith, réf. [2] שxe.

V. L. FITCH, S. L.

MeYer, P. A. Piroue, réf. [3]

A. SCHWAR ZSCHILD,

C. ZuPANCIC, réf. [4]

P. RadVanyi, J. GeNIN, réf. [5]

J. P. AlARD, réf. [6]

J. P. Alard, J. M.

Bolta, A. CordaIL-

LAT, J. Melia, C

Motta, R. Pfohl

A. Sicre, réf. [7]

V. L. Komarov, G.

E. Kozarev, G. V.

SAVChENKo, réf. [9]

A. M. Poskanzer,

G. W. ButLER, E. K.

HYDE, réf. [8]

L. S. Azhgirey, Z.

V. KROMSTEIN, N. K.

Zu, V. I. Petrukhin,

D. M. Khazins, Z.

CiseK, réf. [10]
\end{abstract}

Notre expérience

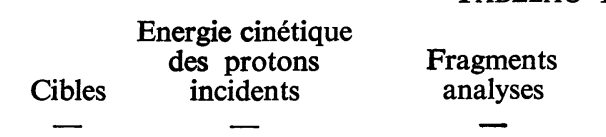

Be, $\mathrm{Pt} \quad 2,9 \mathrm{GeV}$

$\mathrm{Be}, \mathrm{Al} \quad 33 \mathrm{GeV}$

Be, Al, Fe $30 \mathrm{GeV}$

$\mathrm{Be}, \mathrm{Al} \quad 156 \mathrm{MeV}$

$\mathrm{Al}$
(expérience

$220 \mathrm{GeV}$

«E $52 »)$
Li, Be, C $670 \mathrm{MeV}$
U
$5,5 \mathrm{GeV}$
${ }_{2}^{3} \mathrm{He}{ }_{2}^{4} \mathrm{He}$
${ }_{1}^{1} \mathrm{H}{ }_{1}^{2} \mathrm{H}{ }_{1}^{3} \mathrm{H}{ }_{2}^{3} \mathrm{He}{ }_{2}^{4} \mathrm{He}$
Li, Be, B, C

\section{Angle d'éjection (Système L)}

$13^{\circ}-30^{\circ}-60^{\circ}-93^{\circ}$

$13^{\circ}-45^{\circ}-90^{\circ}$

$30^{\circ}$

$13^{\circ}-30^{\circ}$

$15^{\circ}$ à $80^{\circ}$

5 à $40 \mathrm{MeV}$ (pdt) 30 à $120 \mathrm{MeV}(\alpha)$

$5^{\circ} \mathrm{Lab}$.

$20^{\circ}-150^{\circ}$
Energie cinétique
d'éjection

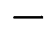

$>$

$>$

$>$

$30 \mathrm{MeV}$ à $156 \mathrm{MeV}$

temps de vol

$E, \mathrm{~d} E / \mathrm{d} x$

\begin{tabular}{|c|c|c|c|c|c|c|}
\hline $\mathrm{C}$ & 670 & $\mathrm{MeV}$ & ${ }_{1}^{2} \mathrm{H}$ & 6,5 Lab. & $500 \mathrm{MeV}$ & temps de vol \\
\hline $\begin{array}{l}\mathrm{C}, \mathrm{Na} \\
\mathrm{l}, \mathrm{Ca}, \mathrm{Fe} \text {, }\end{array}$ & 600 & $\mathrm{MeV}$ & ${ }_{1}^{1} \mathrm{H}{ }_{1}^{2} \mathrm{H}_{1}^{3} \mathrm{H}{ }_{2}^{3} \mathrm{He}{ }_{2}^{4} \mathrm{He}$ & $30^{\circ}-45^{\circ}-75^{\circ}-105^{\circ}$ & $15 \mathrm{MeV} 120 \mathrm{MeV}$ & $\begin{aligned} E, & \mathrm{~d} E / \mathrm{d} x \\
\text { (cet } & \text { article) }\end{aligned}$ \\
\hline & & & $\begin{array}{c}{ }_{1}^{1} \mathrm{H}{ }_{1}^{2} \mathrm{H}{ }_{1}^{3} \mathrm{H}{ }_{2}^{3} \mathrm{He}{ }_{2}^{4} \mathrm{He} \\
\mathrm{Li}, \mathrm{Be}, \mathrm{B}\end{array}$ & $30^{\circ}-45^{\circ}-75^{\circ}$ & $40 \mathrm{MeV}$ & $\begin{array}{c}E, \mathrm{~d} E / \mathrm{d} x \\
\text { (à paraître) }\end{array}$ \\
\hline
\end{tabular}

* CERN, Meyrin-Genève. 
de travaux récents, effectués par divers auteurs dans ce domaine. Peu de résultats expérimentaux existent au voisinage de $600 \mathrm{MeV}$, mis à part les données de Komarov [9] et Azhgirey [10] concernant l'éjection de ${ }_{2}^{3} \mathrm{He}-{ }_{2}^{4} \mathrm{He}$ et ${ }_{1}^{2} \mathrm{H}$ énergétiques émis à très petit angle, ainsi que les données de W. N. Hess et B. J. Moyer [11] à une énergie incidente de $300 \mathrm{MeV}$. Cette dernière expérience concerne uniquement la production de ${ }_{1}^{2} \mathrm{H}$ à un angle de $40^{\circ}$.
De telles mesures présentent un intérêt sur le plan astrophysique pour l'étude de la propagation du rayonnement cosmique galactique. En effet, les rapports ${ }_{1}^{2} \mathrm{H} /{ }_{2}^{4} \mathrm{He},{ }_{2}^{3} \mathrm{He} /{ }_{2}^{4} \mathrm{He}, \mathrm{Li}-\mathrm{Be}-\mathrm{B} / \mathrm{H}$ observés dans le rayonnement cosmique primaire au voisinage de la Terre sont très supérieurs aux valeurs observées dans l'Univers [12], [13]. Le tableau II donne quelques ordres de grandeur concernant ces rapports :

TABLEAU II

$\begin{aligned} & \text { Rapport } \\ & \text { isotopique }\end{aligned}$
${ }_{2}^{3} \mathrm{He} /{ }_{2}^{4} \mathrm{He}$
${ }_{1}^{2} \mathrm{H} /{ }_{2}^{4} \mathrm{He}$
$\frac{\mathrm{LiBeB}}{{ }^{4} \mathrm{He}}$

Il est admis à l'heure actuelle que la composition isotopique $\mathrm{du}$ rayonnement cosmique à la source ne diffère pas sensiblement de la composition isotopique universelle [12], [13], [14]. Les éléments ${ }_{1}^{2} \mathrm{H},{ }_{2}^{3} \mathrm{He}$, $\mathrm{Li}-\mathrm{Be}-\mathrm{B}$, détectés dans le rayonnement primaire au voisinage de la Terre, seraient produits par la fragmentation des noyaux cosmiques lors de leur interaction avec le gaz interstellaire, ce dernier étant composé d'hydrogène $(90 \%)$ et d'hélium $(10 \%)$. Les réactions de fragmentation les plus importantes sont alors les suivantes :

$$
(\alpha+p),(\text { Noyau }+p),(\alpha+\alpha) .
$$

Le premier intérêt est donc de déduire, de l'étude expérimentale de ces réactions, la quantité de gaz interstellaire traversée par le rayonnement primaire.

Un deuxième intérêt est d'étudier les modèles de propagation du rayonnement cosmique dans la Galaxie. Ces modèles [12], [15] tiennent compte de la localisation des sources, ainsi que de la proportion de rayons cosmiques s'échappant de la Galaxie. L'étude complète de ces modèles exige non seulement la connaissance des sections efficaces totales, mais également des rapports isotopiques de production, ainsi que des distributions en énergie des fragments émis [12].

Une étude de la production de $\mathrm{Li}, \mathrm{Be}, \mathrm{B}$ a été effectuée récemment par le groupe de Spectrométrie de Masse d'Orsay [16] et a montré que l'épaisseur de matière traversée était de l'ordre de quelques $\mathrm{g} \times \mathrm{cm}^{-2}$.

En ce qui concerne les isotopes ${ }_{2}^{3} \mathrm{He}$ et ${ }_{1}^{2} \mathrm{H}$, les interactions du type $\left({ }^{4} \mathrm{He}+\mathrm{p}\right)$ ont fait l'objet d'études expérimentales récentes [17], [18] ainsi que les réactions $(\alpha+\alpha)$ [19]. Pour notre part, nous avons porté nos efforts sur les réactions (noyau $+p$ ). Cette étude s'effectue en deux étapes :

1. Analyse de la partie énergétique des Spectres (énergie cinétique d'éjection supérieure à $15 \mathrm{MeV}$ ).

2. Analyse de la partie basse énergie des Spectres

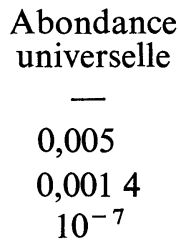

Référence

(énergie cinétique d'éjection comprise entre $2 \mathrm{MeV}$ et $40 \mathrm{MeV}$ ).

Nous décrivons les résultats expérimentaux acquis au cours de la première étape. Le choix de l'énergie incidente de $600 \mathrm{MeV}$ est très favorable, parce que le spectre énergétique du rayonnement cosmique primaire au voisinage de la Terre présente un maximum au voisinage de $500 \mathrm{MeV} /$ nucléon [20].

Une comparaison ultérieure entre les résultats obtenus par les méthodes $\mathrm{Li}-\mathrm{Be}-\mathrm{B} /{ }^{3} \mathrm{He}-{ }^{4} \mathrm{He} /{ }_{1}^{2} \mathrm{H}-{ }_{2}^{4} \mathrm{He}$ devrait permettre d'aboutir à des conclusions astrophysiques intéressantes concernant :

- l'épaisseur de matière interstellaire traversée,

- la nature et la localisation des sources,

- le choix d'un modèle de propagation.

D'autre part, une méthode de datation des météorites utilise la mesure de la quantité $d^{\prime}{ }_{2}^{3} \mathrm{He}$ formée lors de la désintégration de ${ }_{1}^{3} \mathrm{H}$. Une certaine proportion $d^{\prime}{ }_{2}^{3} \mathrm{He}$ est d'origine " spallogénique » (interaction du rayonnement cosmique galactique avec le matériau météoritique) et doit être évaluée grâce à la connaissance des sections efficaces d'éjection de l'ion ${ }_{2}^{3} \mathrm{He}$.

Enfin, sur le plan nucléaire, l'étude expérimentale des distributions angulaires et énergétiques des nuclides légers permet la comparaison avec les prédictions de divers modèles d'éjection : évaporation nucléaire, éjection d'agrégats lors de réactions quasi libres, théorie de Butler et Pearson [6]. L'étude de ces modèles est importante sur le plan astrophysique dans la mesure où elle permet de prévoir les rapports isotopiques à diverses énergies incidentes ou angles d'éjection.

Dans le paragraphe II.3, nous présenterons une comparaison entre nos résultats expérimentaux et un modèle thermodynamique de l'interaction $(\mathrm{p}+$ Noyau $)$.

II. Partie expérimentale. - II. 1 DESCRIPTION DU DISPOSITIF DE DÉTECTION (Fig. 1). - Les cibles solides 


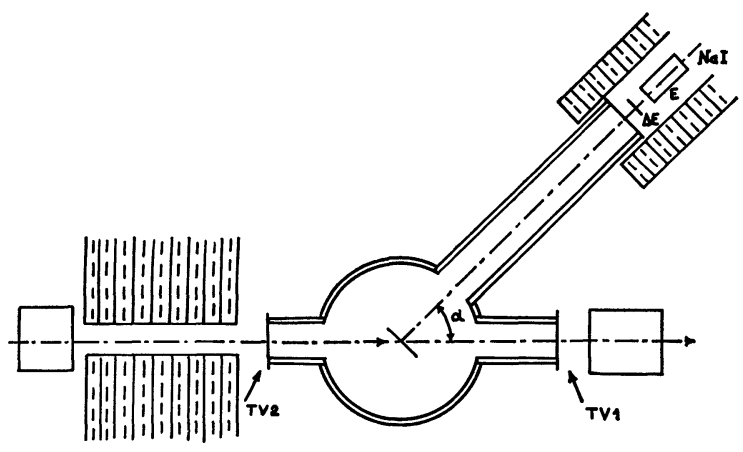

FIG. 1.

sont placées au centre d'une chambre circulaire où est maintenu un vide de l'ordre de $10^{-3} \mathrm{~mm}$ de mercure. La chambre de $50 \mathrm{~cm}$ de diamètre possède des ouvertures latérales permettant de faire varier l'angle de détection des particules. Le faisceau de protons de $600 \mathrm{MeV}$ d'énergie cinétique incidente, dont le flux est d'environ $10^{10}$ particules par seconde, est focalisé au centre de la chambre. Un collimateur en plomb de $50 \mathrm{~cm}$ d'épaisseur permet d'éliminer le halo du faisceau. Deux chambres d'émission secondaire placées de part et d'autre de la chambre à vide permettent la mesure du flux de protons. Le dispositif de détection est constitué d'un télescope $(\Delta E, E)$ composé de la manière suivante :

$\Delta E$ : diode au silicium à barrière de surface d'épaisseur $700 \mu \mathrm{m}$ et de section active $0,5 \mathrm{~cm}^{2}$.

$E$ : scintillateur $\mathrm{NaI}(\mathrm{T} 1)$ cylindrique de diamètre $20 \mathrm{~mm}$ et de longueur $80 \mathrm{~mm}$.

L'ensemble du télescope est placé à l'intérieur d'un blindage en plomb, afin d'éviter le flux de particules parasites en provenance de la salle d'expérience. Le centrage du faisceau s'effectue au moyen de l'observation de deux écrans au sulfure de zinc placés sur les fenêtres d'entrée et de sortie de la chambre à vide.

II. 2 L'ÉLECTRONIQUE ASSOCIÉE. - Le principe d'identification consiste à analyser en coïncidence les signaux fournis par la jonction et le scintillateur lors du passage de la particule. La connaissance de la perte d'énergie $\Delta E$ à travers la diode, donne (par utilisation des tables de G. F. Williamson, J. P. Boujot, J. Picard [21]) l'énergie de la particule. L'électronique associée est décrite sur le schéma synoptique de la figure 2 ; elle permet l'enregistrement sur ruban perforé des couples de valeurs $\Delta E, E$. Une voie « parallèle " reliée à un sélecteur multi-canaux du type TMC contrôle le bon fonctionnement du dispositif. Sur cette voie de contrôle, l'énergie $E$ est fixée au moyen d'un sélecteur monocanal et on analyse, en coïncidence, ses impulsions $\Delta E$ fournies par la jonction. Le traitement des résultats consignés sur rubans s'effectue directement sur ordinateur. La figure 3 montre un spectre d'identification de charge 1 et met en évidence les performances obtenues par cette méthode.

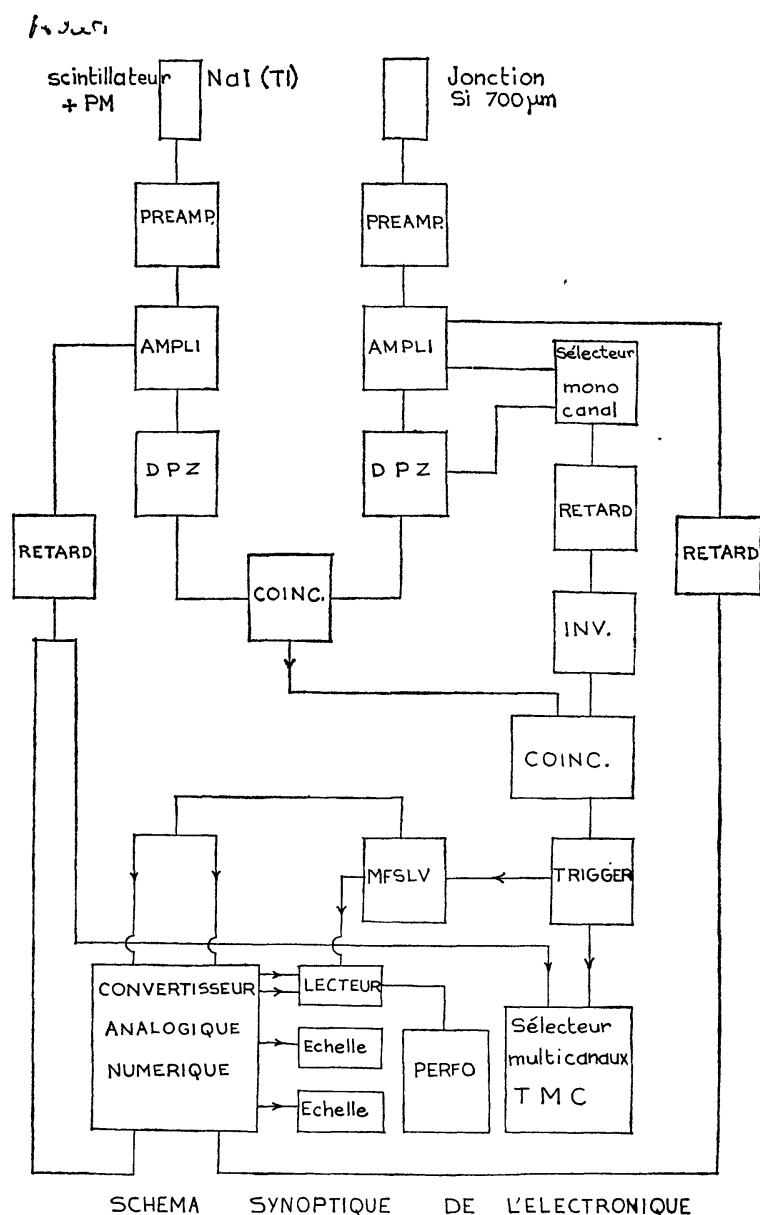

FIG. 2.

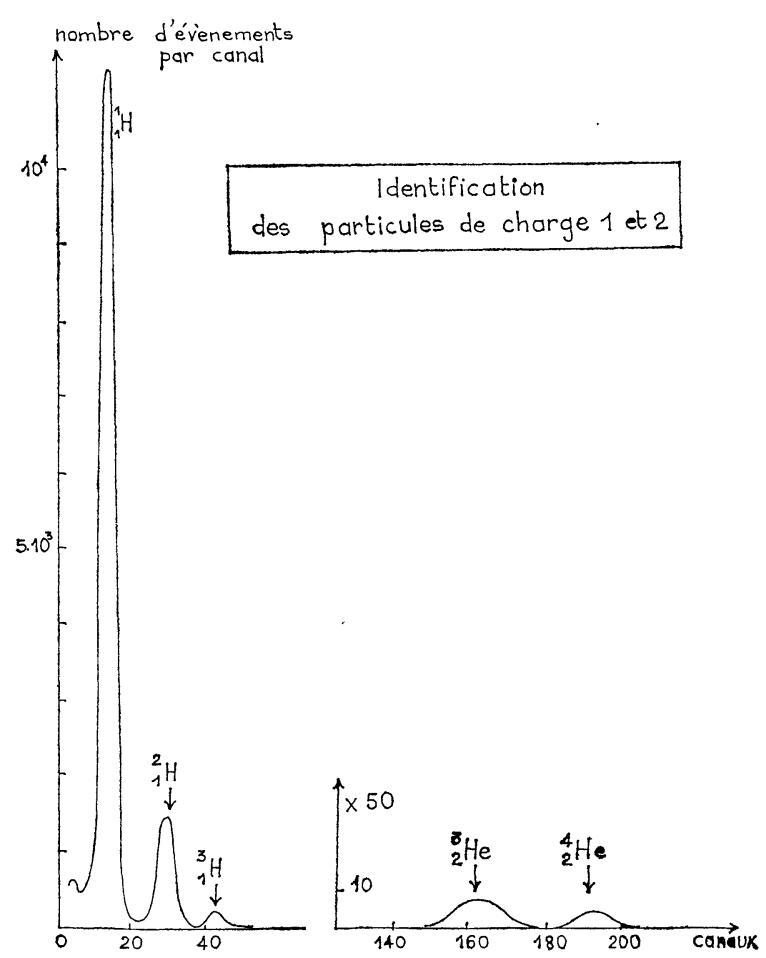

FIG. 3.

II. 3 Résultats EXPéRIMENTAuX. - Les figures 4, 5 , 6, 7 montrent les spectres énergétiques des isotopes 
de charge 1 et 2 produits lors du bombardement d'une cible $d^{\prime 27} \mathrm{Al}$ (d'épaisseur $50 \mu \mathrm{m}$ ) par des protons de $600 \mathrm{MeV}$ à des angles égaux respectivement à $30^{\circ}$, $45^{\circ}, 75^{\circ}, 105^{\circ}$, dans le système du Laboratoire.

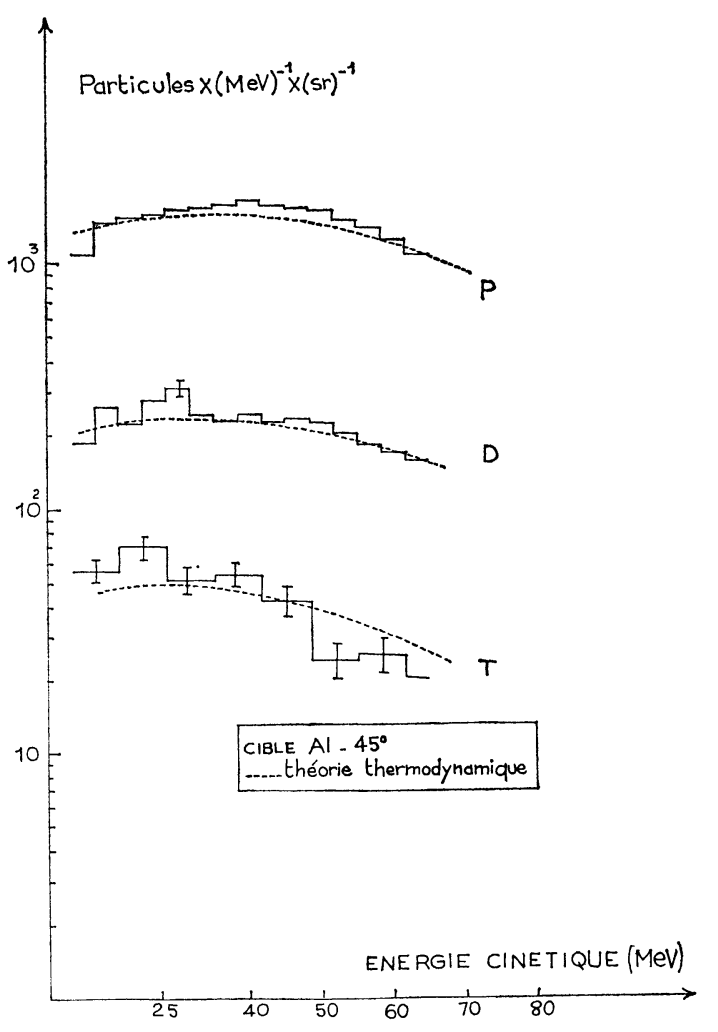

FIG. 4.

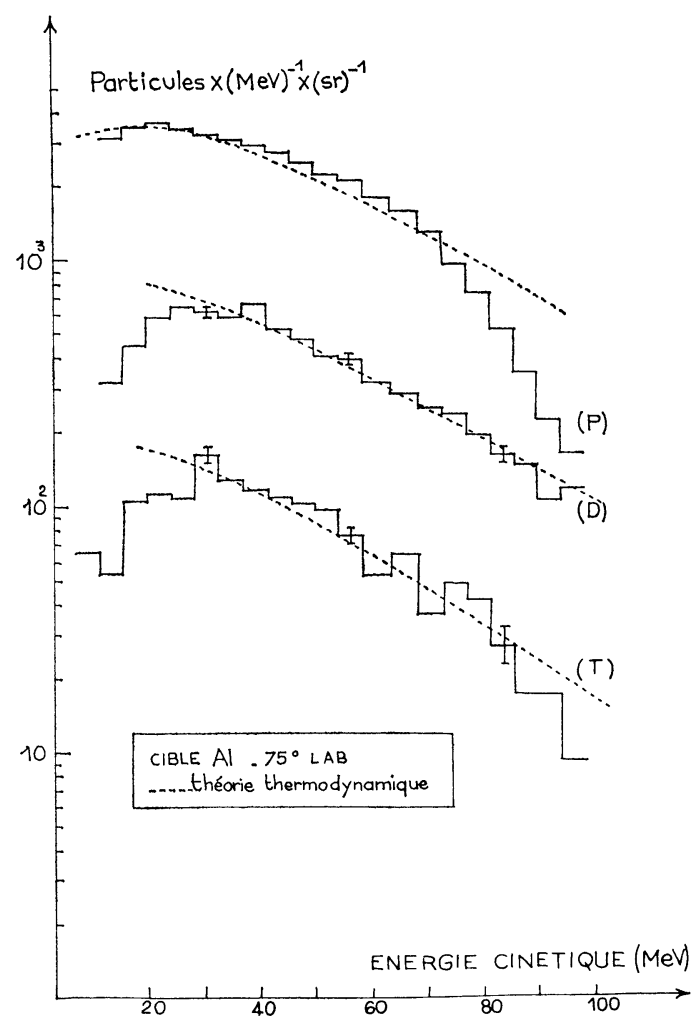

FIG. 5.

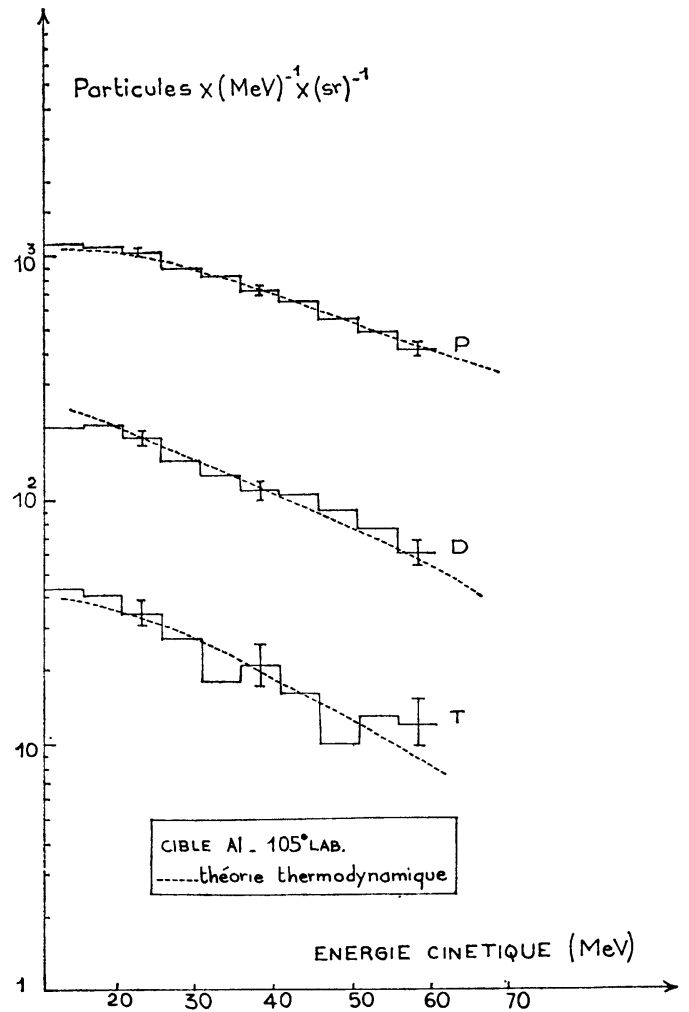

FIG. 6.

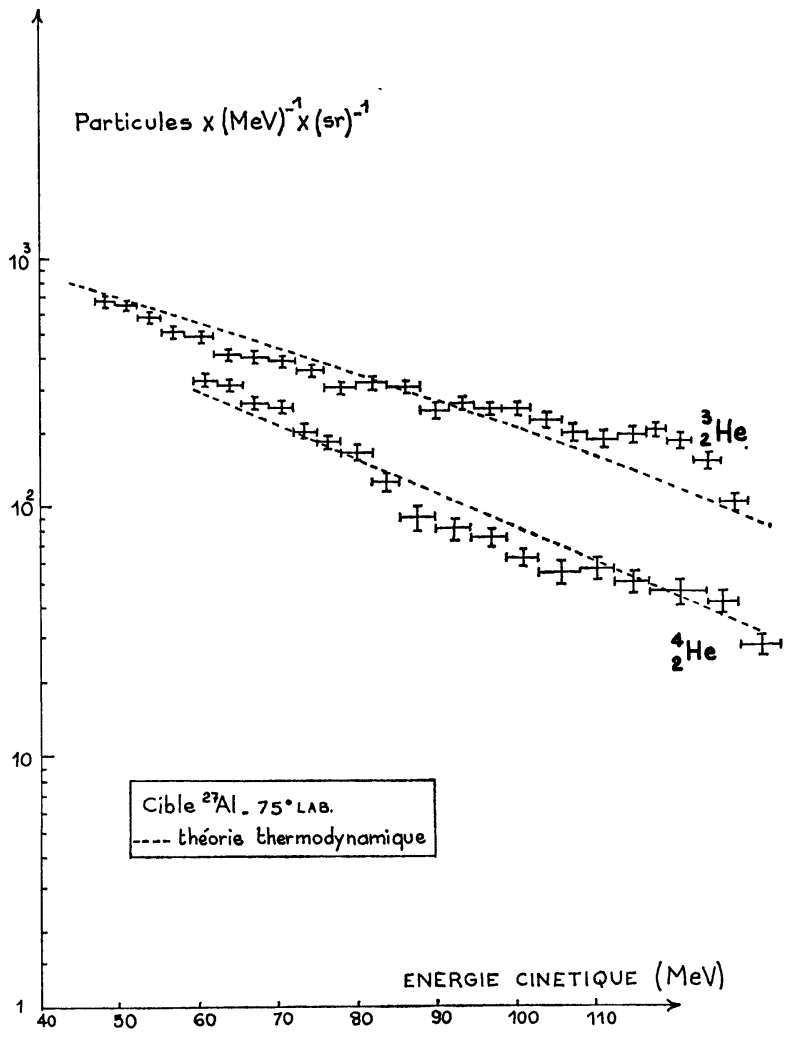

FIG. 7.

Les résultats obtenus avec les cibles de carbone $(250 \mu \mathrm{m})$, d'or $(8 \mu \mathrm{m})$, de fer $(20 \mu \mathrm{m})$, ont été également déterminés. 
Les erreurs indiquées correspondent aux fluctuations statistiques.

En ce qui concerne les spectres énergétiques de particules de charge 2, nous faisons apparaître dans le tableau suivant les valeurs des rapports ${ }_{2}^{3} \mathrm{He} /{ }_{2}^{4} \mathrm{He}$.

\section{TABLEAU III}

\section{Angle}

\begin{tabular}{clcc} 
Nature de la cible & \multicolumn{1}{c}{$45^{\circ}\left(^{*}\right)$} & $75^{\circ}\left(^{*}\right)$ & $105^{\circ}\left(^{* *}\right)$ \\
$-\overline{12 \mathrm{C}}$ & $1,26 \pm 0,04$ & $1,36 \pm 0,03$ & $2,16 \pm 0,15$ \\
${ }^{27 \mathrm{Al}}$ & $1,4 \pm 0,1$ & $1,76 \pm 0,05$ & $1,78 \pm 0,05$ \\
${ }^{197 \mathrm{Au}}$ & $0,481 \pm 0,005$ & $0,92 \pm 0,06$ & $0,695 \pm 0,035$
\end{tabular}

(*) Energie des hélions comprise entre 50 et $100 \mathrm{MeV}$.

(**) Energie des hélions comprise entre 35 et $65 \mathrm{MeV}$.

Ces résultats mettent en évidence la valeur élevée du rapport ${ }_{2}^{3} \mathrm{He} /{ }_{2}^{4} \mathrm{He}$ dans la partie énergétique du spectre, notamment en ce qui concerne les noyaux légers $\left({ }^{12} \mathrm{C},{ }^{27} \mathrm{Al}\right)$. Ce dernier point est important en astrophysique, le noyau ${ }^{12} \mathrm{C}$ faisant partie du groupe (CNO) qui présente un maximum dans le spectre des abondances cosmiques [22]. Ces résultats, qui complètent des résultats partiels obtenus précédemment [23], sont en accord avec les données expérimentales présentées par A. M. Poskanzer [8], à une énergie incidente de $5,5 \mathrm{GeV} / \mathrm{c}$, donc nettement supérieure à l'énergie la plus probable du spectre du rayonnement cosmique primaire.

III. Aspect théorique. - Afin d'évaluer les taux de production de fragments légers rapides dans les interactions de protons énergétiques avec les noyaux, nous proposons une théorie thermodynamique de l'interaction primaire, inspirée de la méthode de calcul proposée par R. Hagedorn [24], [25] dans le cas des interactions hadron-hadron.

Notre modèle diffère de celui d'Hagedorn par les points suivants :

$1^{0}$ Il s'agit d'une description statistique d'un processus de cascade nucléaire associé à l'éjection d'agrégats de masse $2,3,4 \ldots$

$2^{\circ} \mathrm{La}$ 《Température » utilisée pour décrire ce processus est une température moyenne; nous ne connaissons pas pour l'instant la relation entre la température et la vitesse des particules de cascade.

$3^{\circ} \mathrm{La}$ fonction de partition utilisée pour décrire l'interaction contient la présence de groupements de nucléons.

Le «temps de relaxation" $\tau$ caractérisant le processus peut être évalué de la manière suivante [26] : si $T$ est la température moyenne, $k$ la constante de Boltzmann et $\hbar$ la constante de Planck :

$$
\tau \simeq \frac{\hbar}{2 k T} \text {. }
$$

Pour une température $T$ de l'ordre de $100 \mathrm{MeV}$, on trouve :

$$
\tau \# 0,32 \times 10^{-23} \text { seconde. }
$$

Dans le cas du noyau ${ }^{27} \mathrm{Al}$, la durée $t_{\mathrm{c}}$ de la cascade nucléonique est de l'ordre de $2,5 \times 10^{-23}$ seconde ; dans le cas du noyau ${ }^{197} \mathrm{Au}$, elle est de !l'ordre de $5 \times 10^{-23}$ seconde. On a donc dans le cas de ${ }^{197} \mathrm{Au}$ :

$$
\left(\frac{\tau}{t_{\mathrm{c}}}\right)_{\mathrm{Au}} \# 0,06 \text {. }
$$

Il en résulte que le temps de relaxation est inférieur au temps de développement de la cascade nucléonique, ce qui nous permet de traiter chaque élément de volume comme étant en équilibre thermodynamique.

- Notations utilisées :

$\gamma_{0}$ : facteur de Lorentz de la particule incidente dans le système du centre de masse protonnoyau :

$$
\gamma_{0}=\left(1-\beta_{0}^{2}\right)^{-1 / 2},
$$

$\gamma$ : facteur de Lorentz correspondant à la composante longitudinale de la vitesse d'un élément de volume de matière nucléaire :

$$
\gamma=\left(1-\beta^{2}\right)^{-1 / 2},
$$

$\lambda$ : variable de vitesse définie par:

$$
\lambda=\operatorname{sgn}(\beta) \frac{\gamma-1}{\gamma_{0}-1} \text {. }
$$

Nous pouvons décrire le spectre énergétique des particules éjectées dans le système du centre de masse par :

$$
W_{\mathrm{i}}(\mathbf{p})=\int_{-1}^{+1} F\left(\gamma_{0}, \lambda\right) \mathfrak{L}(\beta) f_{\mathrm{i}}\left(\varepsilon^{\prime}, T\right) \mathrm{d} \lambda .
$$

$F\left(\gamma_{0}, \lambda\right)$ est la distribution de vitesse des particules de cascade, $f_{\mathrm{i}}\left(\varepsilon^{\prime}, T\right)$ est le spectre énergétique des particules ayant la variable de vitesse $\lambda$.

$\varepsilon^{\prime}=\left(p^{2}+m^{2}\right)^{1 / 2}$ est l'énergie' totale dans le système $\left(K^{\prime}\right)$ défini par $\lambda=0$.

$\mathfrak{L}(\beta)$ est l'opérateur transformée de Lorentz permettant le passage du système $\left(K^{\prime}\right)$ au système du centre de masse $(K)$

$F\left(\gamma_{0}, \lambda\right)=\frac{1}{V \sigma \Delta t} \int u(\lambda, \mathbf{x}, t, \rho) 2 \pi \rho \mathrm{d} \rho d^{3} \times \mathrm{d} t$.

$u(\lambda, \mathbf{x}, t, \rho)$ représente le nombre de particules de cascade émises au temps $t$ à la coordonnée $\mathbf{x}$ avec un paramètre d'impact $\rho$

$V$ : volume d'interaction

$\sigma=4 \pi R_{0}^{2}\left(R_{0}=\right.$ rayon nucléaire $)$.

La fonction $F\left(\gamma_{0}, \lambda\right)$ a été évaluée par J. P. Viallefont et J. C. Tamain, à l'aide d'un calcul de MonteCarlo décrivant la cascade nucléonique à haute énergie [28].

L'expression de $f_{\mathrm{i}}\left(\varepsilon^{\prime}, T\right)$ décrivant les spectres énergétiques est obtenue en calculant la «grande fonstion 
de partition " $Z$ tenant compte de la formation de ${ }_{1}^{1} \mathrm{H},{ }_{1}^{2} \mathrm{H},{ }_{1}^{3} \mathrm{H},{ }_{2}^{3} \mathrm{He},{ }_{2}^{4} \mathrm{He}$ :

$\log (1+Z)=$

$=\frac{V}{2 \pi^{2}} \int_{0}^{\infty} p^{2} \mathrm{~d} p \sum_{i=1}^{A} g_{1} \sum_{n=1}^{\infty} \frac{(-1)^{l(n+1)} z^{n l} X_{p l}^{n}}{n}$

avec

$$
X_{p l}=\exp \left[-\frac{1}{T}\left(p^{2}+l^{2} m^{2}\right)^{1 / 2}\right]
$$

et

$$
z^{l}=\exp \left(\frac{\mu_{l}}{T}\right)
$$

$g_{l}$ tient compte de l'existence possible d'isobares (exemple : ${ }_{1}^{3} \mathrm{H}$ et ${ }_{2}^{3} \mathrm{He}$ ).

On aboutit alors à :

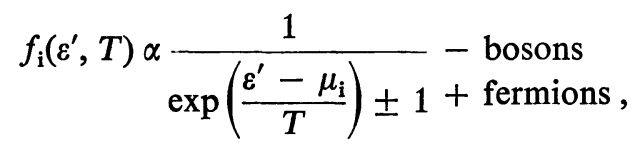

$\mu_{\mathbf{i}}$ est le «potentiel chimique » associé à chacune des particules émises. L'intégration numérique de l'expression (4) conduit aux distributions doublement différentielles $\mathrm{d}^{2} N_{\mathrm{i}} / \mathrm{d} \Omega . \mathrm{d} p$ d'éjection de particules à partir $\mathrm{du}$ noyau.

IV. Comparaison entre résultats théoriques et expérimentaux. - Les résultats déduits de l'application de la thermodynamique statistique sont reportés sur les figures $2,3,4,5$ en traits pointillés. Nous remarquons d'une part que l'allure des spectres expérimentaux est bien décrite par notre formalisme théorique et que, d'autre part, les rapports entre les isotopes observés expérimentalement sont bien ceux prévus par la théorie, tous les spectres étant normalisés par rapport aux spectres expérimentaux de protons.

Sur la figure 7 , on observe en particulier la valeur élevée du rapport ${ }^{3} \mathrm{He} /{ }^{4} \mathrm{He}$ dans la bande d'énergie 50-120 MeV. Sur le plan expérimental, nous déterminons actuellement les spectres des ${ }^{3} \mathrm{He}$ et ${ }^{4} \mathrm{He}$ d'énergie inférieure à $50 \mathrm{MeV}$, de manière à préciser l'importance de ce phénomène pour les noyaux légers tels que ${ }^{12} \mathrm{C},{ }^{27} \mathrm{Al}$.

Conclusion. - Les résultats expérimentaux obtenus nous ont donné la possibilité d'étudier la production de nuclides légers à divers angles et avec des cibles variées. Nous soulignons la variation du rapport ${ }_{2}^{3} \mathrm{He} /{ }_{2}^{4} \mathrm{He}$ en fonction du nombre de masse du noyaucible. En particulier, le rapport ${ }^{3} \mathrm{He} /{ }^{4} \mathrm{He}$ est élevé dans le cas des noyaux légers $(\mathrm{C}, \mathrm{Al})$ et il devient nécessaire, pour ces noyaux, de déterminer l'ensemble du spectre énergétique. Ce dernier point est actuellement en cours d'étude au CERN. L'intérêt astrophysique d'un tel résultat est évident, dans la mesure où les noyaux légers du type $\mathrm{C}-\mathrm{N}-\mathrm{O}-$ se traduisent par un pic abondant dans le spectre des abondances cosmiques.

En ce qui concerne la production de ${ }_{1}^{2} \mathrm{H}$ et ${ }_{1}^{3} \mathrm{H}$, les rapports $d / p, t / d$ sont de l'ordre de 0,2 quels que soient l'énergie ou l'angle d'éjection. La comparaison de ces résultats avec ceux obtenus à des énergies incidentes différentes - notamment A. M. Poskanzer [7] montre que les rapports $d / p, t / d$ sont également peu sensibles à la variation de l'énergie incidente. Ceci amène une simplification importante dans le traitement de la propagation du rayonnement cosmique galactique.

Enfin, nous avons montré qu'un modèle thermodynamique relativement simple permet d'interpréter les spectres obtenus et de prévoir les rapports $d / p, t / d$, ${ }^{3} \mathrm{He} /{ }^{4} \mathrm{He}$ à divers angles ou énergies d'éjection.

Ce dernier point est utilisable directement dans l'étude astrophysique. Sur le plan nucléaire, cette méthode de calcul met en jeu un processus statistique d'agglomération de nucléons et n'est pas en contradiction avec le mécanisme de Butler et Pearson. Toutefois, dans sa forme actuelle, la théorie de Butler et Pearson ne permet pas de prévoir les rapports de production - ainsi que l'ont du reste montré Piroué et Smith lors de leurs expériences à une énergie incidente de $2,8 \mathrm{GeV}$ [2].

Actuellement, nous poursuivons au CERN l'expérience SC 33 par une spectrométrie "basse énergie » des $p, d, t,{ }^{3} \mathrm{He},{ }^{4} \mathrm{He}$ (énergie des fragments : de $3 \mathrm{MeV}$ à $40 \mathrm{MeV}$ ). Les cibles utilisées sont $\mathrm{Be}, \mathrm{C}$, $\mathrm{Na}, \mathrm{Al}, \mathrm{Ca}, \mathrm{Fe}, \mathrm{Au}$ et les angles : $30^{\circ}-45^{\circ}-75^{\circ}-105^{\circ}$. Les résultats complets ainsi que l'interprétation de l'ensemble de cette expérience seront donnés dans une prochaine publication.

Nous remercions particulièrement Messieurs les Professeurs P. Cuër, R. Hagedorn et H. Reeves pour leurs conseils sur les plans théorique et astrophysique, M. P. Siffert, du PREN de Strasbourg, pour la réalisation des jonctions nécessaires à cette expérience, ainsi que M. R. Boudeaud du Centre de Calcul de Clermont-Ferrand, pour son aide efficace lors de l'exploitation sur ordinateur.

\section{Bibliographie}

[1] Alard (J. P.), CERN PH III 70/41 ; PH III 70/51 ; PH III $71 / 28$.

[2] Piroue (P. A.), Smith (A. J. S.), Phys. Rev., 1966, 148, 1315.
[3] Fitch (V. L.), Meyer (S. L.), Piroue (P. A.), Phys. Rev., 1962, 126, 1849.

[4] Schwarzschild (A.), Zupancic (C.), Phys. Rev., $1963,129,854$. 
[5] Radvanyi (P.), Genin (J.), J. Phys. Rad., 1960, 21, 322.

[6] Alard (J. P.), Thèse, Clermont-Ferrand, 1971, n ${ }^{\circ} 145$.

[7] Alard (J. P.), Bolta (J. M.), Cordaillat (A.), Melia (J.), Pfohl (R.), Sicre (A.), C. R. Acad. Sci. Paris, 1969, 269, 212.

[8] Poskanzer (A. M.), Butler (G. W.), Hyde (E. K.), Phys. Rev., 1971, C 3, 882.

[9] Komarov (V. L.), Kozyrev (G. E.), SAvchenko (G. V.), Reprint JINR, Dubna, 1969, P 14373. Reprint JINR Dubna, 1969, P, 1-4227.

[10] Azhgirey (L. S.), Krumsten (Z. V.), Zu (N. K.), Petrukhin (V. I.), Kazins (D. M.), CizeK (Z.).

[11] Hess (W. N.), Moyer (B. J.), Preprint Dubna, 1970, P 1-4985.

[12] Ginzburg (V. L.), Syrovatski (S. I.), « The Origin of Cosmic Rays » Pergamon Press, OxfordLondon, 1964.

[13] Meyer (J. P.), HaAge (D. E.), Mc Donald (F. B.), Can. J. Phys., 46, S. 503.

[14] Koch (L.), BIST CEA, 1968, no 132.

[15] ReEves (H.), Internat School of Physics « Enrico Fermi », July 1971, L III course, Lect. V.
[16] Yiou (F.), Ann. Phys., 1968, 3, 1969.

[17] Gardes (J.), Thèse $3^{\mathrm{e}}$ cycle, Clermont-Ferrand, 1971.

[18] Kozodaev, Kulyukin, Sulyaev, Filippov, ScherBAKOV, JETP, $12, \mathrm{n}^{\circ} 4,644$.

[19] JuNG (M.), Thèse, Strasbourg, 1971.

[20] Webber (W. R.), Handb. of Physics Vol. XLVI/2.

[21] Williamson (C. F. ), Boujot (J. P.), PiCard (J.), Rapp. CEA, 1966, R-3042.

[22] Suess (H. E.), Urey (H. C.), Rev. Mod. Phys., 1956, $28,53$.

[23] Alard (J. P.), Cordaillat (A.), Cornago (M.), Fargeix (J.), Fraysse (L.), Roche (G.) et Sicre (A.), C. R. Acad. Sci. Paris, 1970, 270, 1438.

[24] HAgEdoRN, CERN. 67/269/5. TH 751.

[25] Hagedorn (R.), RANFT (J.), Suppl. Nuovo. Cim., 1968, 6, 298.

[26] Frey, Laigle (P.) et Salmon (J.), Revue Générale de Thermique $\mathrm{n}^{0} 105$, sept. 1970.

[27] Viallefont (P.), Thèse $3^{\mathrm{e}}$ cycle, Clermont-Ferrand, 1970.

[28] Tamain (J. C.), Thèse $3^{\mathrm{e}}$ cycle, Clermont-Ferrand, 1971. 